\title{
The effects of hyperosmolal coronary perfusion on the haemodynamic, metabolic and ultrastructural changes of myocardial anoxia*
}

\author{
NORMAN BRACHFELD \\ M.D. \\ JAMES Christodoulou \\ M.D. \\ ROBERT ERLANDSON \\ Ph.D. \\ Charles Smithen \\ M.D. \\ Department of Medicine, The New York Hospital-Cornell Medical Center and the \\ Department of Pathology, Memorial Hospital, New York, New York 10021
}

\begin{abstract}
Summary
Recovery from anoxia has been evaluated in the isovolumic non-recirculating paced perfused rat heart. Seventy studies were performed consisting of $15 \mathrm{~min}$ of aerobic perfusion (AP); $\mathbf{A P}+\mathbf{1 5}$ min anoxic perfusion; and $A P+15$ min anoxic perfusion +15 min reoxygenation (recovery). Krebs-Ringer-bicarbonate + $5 \mathrm{mmol}$ glucose (KRB) (290 mmol) was compared to KRB + mannitol (350 mmol). Mannitol decreased myocardial water content. It improved recovery of haemodynamic function after reoxygenation. With KRB alone left ventricular systolic peak pressure (LVSP) decreased $32 \%$ and maximum $\mathrm{dP} / \mathrm{dt}$ by $50 \%$. With mannitol added LVSP decreased $18 \%$ and $\mathrm{dP} / \mathrm{dt}$ $21 \%(P<0.01)$. KRB and mannitol did not differentially affect total coronary flow, lactate and glucose extraction, tissue glycogen, creatine phosphate or adenine nucleotide concentrations.

No difference in submicroscopic appearance was noted with either perfusate during aerobic perfusion. Anoxic hearts perfused with isosmolal KRB demonstrated the most severe ultrastructural alterations including mitochondrial swelling with disruption of cristae and extraction of matrix components, myofibrillar fusion and contraction bands, and subsarcolemmal oedema and vacuolization. These changes were only partially reversed during reoxygenated perfusion. However, cellular changes were reversed or markedly improved during both the anoxic and reoxygenation perfusion periods with hyperosmolal
\end{abstract}

* Supported by: USPHS Contract No. N01 HV 71439; The Lincoln National Life Ins. Co.; The Manhattan Life Ins. Co.; The Prudential Ins. Co. of America and North American Reassurance Co.

Address for reprints: Dr Norman Brachfeld, The New York Hospital, 525 East 68th Street, New York, New York 10021 . solutions. When studied by silicone rubber injection of the microcirculation, only focal capillary endothelial cell swelling was noted and no difference in arteriolar or capillary filling was observed with either perfusate. Mannitol appears to improve $L V$ function by direct myocardial osmotic action unrelated to enhanced energy production.

\section{Introduction}

Both ischaemia and anoxia have been shown to induce a significant degree of tissue swelling. The physiological significance of such changes may be expressed with particularly detrimental effects in myocardium. Myocardial cellular swelling may dilute intracellular contents and lead to palpable firmness of the ventricular wall. Reduction in ventricular compliance caused by high membrane tensions can seriously impair haemodynamic performance. Recent studies by Willerson et al. (1972) demonstrated the beneficial action of elevated osmolality in protecting the ischaemic myocardium against the potentially toxic effects of increased cell volume. Its mechanism of action, however, is not completely understood. These studies were designed to evaluate the effects of a hyperosmolal agent (mannitol) during anoxia under the controlled conditions provided by the isolated perfused non-recirculating Langendorff rat heart preparation. Haemodynamic, metabolic, optical and electron microscopic and latex vascular injection studies were performed to explore its mechanism of action. The results of haemodynamic and metabolic studies form the subject of this preliminary report.

\section{Methods}

Seventy isolated rat hearts were perfused in a tandem Langendorff apparatus modified to permit rapid exchange of perfusates varied as to osmolality 


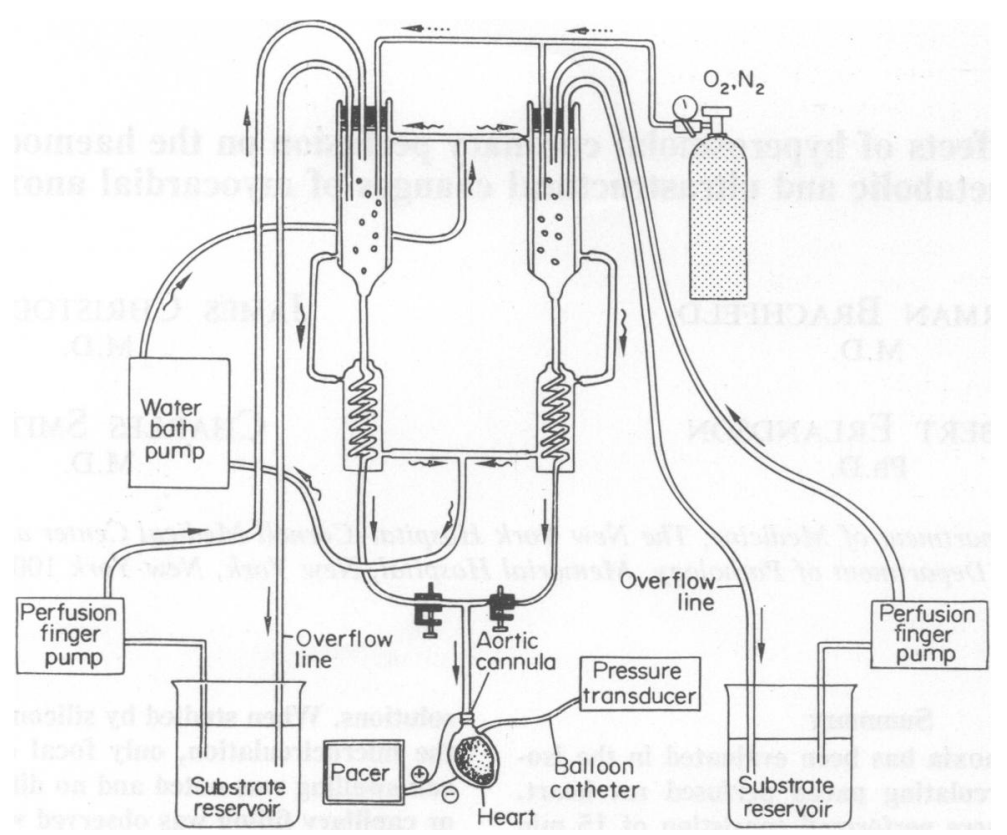

Fig. 1. Modified Langendorff rat heart perfusion apparatus. A retrograde, non-circulating tandem system is illustrated allowing for ventricular pressure recording during isovolumic ventricular contraction by inflation of a balloon catheter attached to a pressure transducer, and control of heart rate by an external battery powered pacemaker.

and $p \mathrm{O}_{2}$ (Fig. 1). Male, fed, albino rats of the Sherman strain weighing 250-290 g were used. Rats were killed by axial fracture, the heart rapidly removed and mounted on the aortic cannula. The procedure required $40 \mathrm{sec}$ from sacrifice to onset of perfusion. The substrate reservoir was set to deliver a perfusion pressure of $75 \mathrm{mmHg}$ and buffer was maintained at $37^{\circ} \mathrm{C}$ and a $\mathrm{pH}$ of $7 \cdot 4$. After onset of perfusion, a small latex balloon catheter was inserted into the left ventricle. The balloon was inflated (diastolic pressure $=$ atmospheric pressure) and ventricular pressure and its first derivative recorded. A stab wound in the wall of the left ventricle prevented accumulation of thebesian drainage. Heart rate was maintained at a constant of 300 beats/min during aerobic perfusion by bipolar pacing. Coronary flow was measured by collection of fluid ejected via the pulmonary artery. This venous efflux was assayed as described below. Arterial samples were drawn from the perfusion cannula.

\section{Perfusates}

The control perfusate consisted of modified KrebsRinger bicarbonate buffer containing $5 \mathrm{mmol}$ glucose, $0.1 \mathrm{~mm} \mathrm{Na}$ Lactate and $25 \mathrm{mu}$ of glucagon free insulin $/ \mathrm{ml}$ solution. Its osmolality was 290 mmol. The experimental perfusate differed by the addition of $20 \%$ mannitol sufficient to raise the osmolality of the solution to $350 \mathrm{mmol}$.

During aerobic studies perfusate $\mathrm{pO}_{2}$ was maintained at $550 \pm 10 \mathrm{mmHg}$ by bubbling with $95 \%$ $\mathrm{O}_{2}+5 \% \mathrm{CO}_{2}$. For anoxic studies, solutions were bubbled with $95 \% \quad \mathrm{~N}_{2}+5 \% \quad \mathrm{CO}_{2}$ and $p \mathrm{O}_{2}$ was $50 \pm 5 \mathrm{mmHg}$.

\section{Experimental design}

The two perfusates were compared during three sequential study periods: (a) $15 \mathrm{~min}$ of paced aerobic perfusion (control), (b) $15 \mathrm{~min}$ of anoxic perfusion (anoxia), and (c) $15 \mathrm{~min}$ of paced aerobic perfusion (recovery). Metabolic, haemodynamic and histological studies were performed on a series of fourteen hearts following each of the three periods. An additional group of seven hearts was perfused with isosmolal buffer during control and anoxic periods and with the hyperosmolal solution during the recovery phase.

\section{Assay procedures}

The arterio-venous concentration differences of 
buffer samples were multiplied by coronary flow to express substrate consumption/production. Glucose and lactate assays were performed in triplicate with a coefficient of variation of $\pm 2 \%$ by automated techniques previously described (Apstein, Puchner and Brachfeld, 1970). At the termination of each perfusion period, hearts were compressed by Wollenberger clamps taken from liquid nitrogen. Frozen specimens were finely ground and wet/dry weight determined after drying of an aliquot at $105^{\circ} \mathrm{C}$ for $24 \mathrm{hr}$. An additional aliquot was weighed in a tared frozen centrifuge tube and homogenized with $\mathrm{HClO}_{4}$. A sample of the supernatant was extracted with $\mathrm{KOH}$ for glycogen determination. The remainder was assayed enzymatically for ATP, creatine phosphate, ADP and AMP.

\section{Electron microscopic studies}

In a separate series of fourteen hearts the cardiac apex was excised during perfusion and was immediately immersed in s-collidine buffered $2 \%$ acrolein and $5 \%$ glutaraldehyde fixative for $1 \cdot 5-2 \mathrm{hr}$. The tissue was then rinsed in buffer and postfixed for $1 \mathrm{hr}$ in $1 \%$ s-collidine buffered osmium tetroxide. After dehydration, specimens were embedded in Maraglas-D.E.R. 732 epoxy resin. Thin sections were stained with uranyl acetate followed by lead citrate and were examined in a Siemens Elmiskop 101 microscope. Thick $(1 \mu)$ sections for light microscopic examination were stained with toluidine blue.

\section{Microcirculation studies}

A silicone rubber injection mass was prepared so as to attain a viscosity of 15-25 centipoise after catalysation. The material was introduced at a fixed perfusion pressure identical with that used during buffer perfusion. The mixture was admitted to the retrograde aortic perfusion cannula by a system designed to exclude the interposition of air bubbles.

Hearts continued to beat for 2-3 min and perfusion pressure was maintained until the injection mass appeared in the venous drainage. The heart was then removed from the cannula and allowed to cure overnight at room temperature. Curing took place with non-exothermic cross-linking and minimum volume change. The tissue was then dehydrated by immersion in ethyl alcohol of progressively increasing concentration. It was cleared and rendered transparent by immersion in synthetic methyl salicylate.

Sections of 200-300 $\mu$ were prepared for direct microscopic examination and also cut at $20 \mu$ for routine HE staining.

\section{Results \\ Haemodynamics \\ During control aerobic perfusion, hyperosmolality}

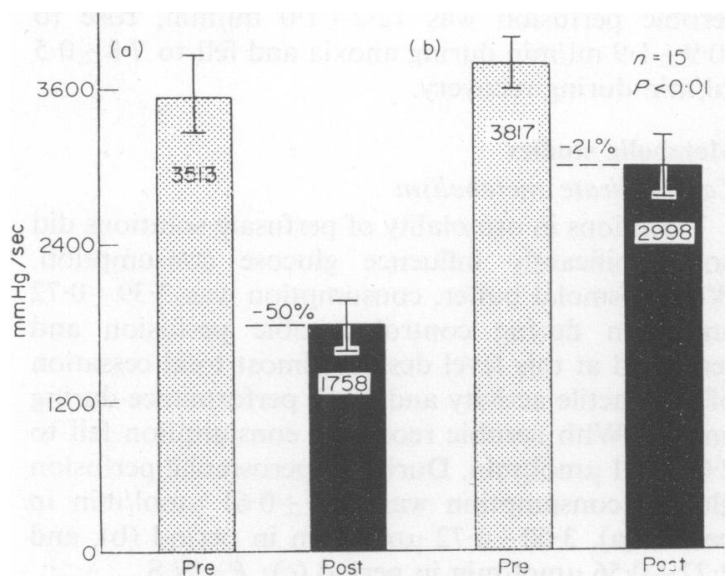

FIG. 2. Effect of mannitol on left ventricular maximum $\mathrm{dP} / \mathrm{dt}$. Enhanced ventricular performance during the reoxygenated recovery period ('post' $=$ after anoxia) was noted with mannitol. (a) KRB; (b) KRB and mannitol.

did not significantly affect peak left ventricular systolic pressure. Active contraction ceased soon after onset of anoxia and pressures could not be recorded. During aerobic recovery neither series of hearts reached pre-anoxic performance levels. Nevertheless, those perfused with the hyperosmolal solution showed a significant improvement over those perfused with KRB alone. Isosmolal perfusate pressures reached $68 \%$ of aerobic controls $(127 \pm 5$ $\rightarrow 86 \pm 6 \mathrm{mmHg}$ ); hyperosmolal pressures reached $77 \%$ of control measurements $(132 \pm 5 \mathrm{mmHg} \rightarrow$ $102 \pm 7 \mathrm{mmHg}) P=<0 \cdot 01$. Enhanced performance was more evident when the first derivative of the ventricular pressure curve was analysed (Fig. 2). With KRB buffer alone, mean recovery values of $\mathrm{dP} / \mathrm{dt}$ were $50 \%$ of control $(3513 \pm 328 \mathrm{mmHg} / \mathrm{sec}$ $\rightarrow 1758 \pm 172 \mathrm{mmHg} / \mathrm{sec}$ ). Perfusion with hyperosmolal solution enhanced performance to $79 \%$ of control aerobic perfusion $(3817 \pm 215 \rightarrow 2998 \pm 234$ $\mathrm{mmHg} / \mathrm{sec}) \boldsymbol{P}=<0.01$. Improved recovery was also demonstrable when hyperosmolality was induced at the termination of the anoxic period. Under these circumstances, peak systolic recovery was $86 \%$ and $\mathrm{LV} \mathrm{dP} / \mathrm{dt} 81 \%$ of aerobic controls.

\section{Coronary flow}

Coronary flow during perfusion with isosmolal buffer was $11.9 \pm 0.7 \mathrm{ml} / \mathrm{min}$, rose markedly at the onset of anoxia to reach $19 \cdot 2 \pm 2 \cdot 4 \mathrm{ml} / \mathrm{min}$ and fell to $8.4 \pm 0.9 \mathrm{ml} / \mathrm{min}$ with cessation of contractile activity. The mean level during recovery was $7 \cdot 12 \pm$ $0.6 \mathrm{ml} / \mathrm{min}$, a flow rate that did not differ significantly from aerobic control when flow was corrected for contractile activity. Hyperosmolal perfusion did not significantly change these rates. Coronary flow during 
aerobic perfusion was $12.2 \pm 1.0 \mathrm{ml} / \mathrm{min}$, rose to $20.9 \pm 1.9 \mathrm{ml} / \mathrm{min}$ during anoxia and fell to $7.8 \pm 0.5$ $\mathrm{ml} / \mathrm{min}$ during recovery.

\section{Metabolic studies}

\section{Carbohydrate metabolism}

Variations in osmolality of perfusate solutions did not significantly influence glucose consumption. With isosmolal buffer, consumption was $3.39 \pm 0.72$ $\mu \mathrm{mol} / \mathrm{min}$ during control aerobic perfusion and remained at this level despite almost total cessation of contractile activity and work performance during anoxia. With aerobic recovery, consumption fell to $2.00 \pm 1 \cdot 1 \mu \mathrm{mol} / \mathrm{min}$. During hyperosmolal perfusion glucose consumption was $3.28 \pm 0.61 \mu \mathrm{mol} / \mathrm{min}$ in period (a), $3.00 \pm 0.72 \mu \mathrm{mol} / \mathrm{min}$ in period (b), and $2.22 \pm 0.56 \mu \mathrm{mol} / \mathrm{min}$ in period (c); $P=$ N.S.

Mean lactate production during aerobic isosmolal perfusion with KRB buffer was $0.57 \pm 0.16 \mu \mathrm{mol} /$ min. Production increased to $3.05 \pm 0.53 \mu \mathrm{mol} / \mathrm{min}$ during anoxic perfusion. During recovery, production fell markedly and did not differ significantly from aerobic control, $0 \cdot 78 \pm 0 \cdot 13 \mu \mathrm{mol} / \mathrm{min}$. The use of the hyperosmolal perfusate did not alter lactate metabolism at any phase of the study. Production was $0.81 \pm 0.12 \mu \mathrm{mol} / \mathrm{min}$ in period (a), $3.04 \pm 0.26$ $\mu \mathrm{mol} / \mathrm{min}$ in period (b), and $1.07 \pm 0.13 \mu \mathrm{mol} / \mathrm{min}$ in period (c); $P=$ N.S.

Tissue glycogen concentration was $103 \pm 11$ $\mu \mathrm{mol} / \mathrm{g}$ dry wt during aerobic perfusion with isosmolal buffer. It fell to $41 \pm 5 \mu \mathrm{mol} / \mathrm{g}$ dry wt during anoxic perfusion and remained close to this level during aerobic recovery $(43 \pm 4 \mu \mathrm{mol} / \mathrm{g}$ dry $w \mathrm{t})$ (Fig. 3). Hyperosmolal tissue glycogen levels were $93 \pm 10 \mu \mathrm{mol} / \mathrm{g}$ dry wt during period (a), $37 \pm 1$ $\mu \mathrm{mol} / \mathrm{g}$ dry wt during period (b), and $47 \cdot 7 \pm 7 \mu \mathrm{mol} / \mathrm{g}$ dry wt during period (c); $P=$ N.S.

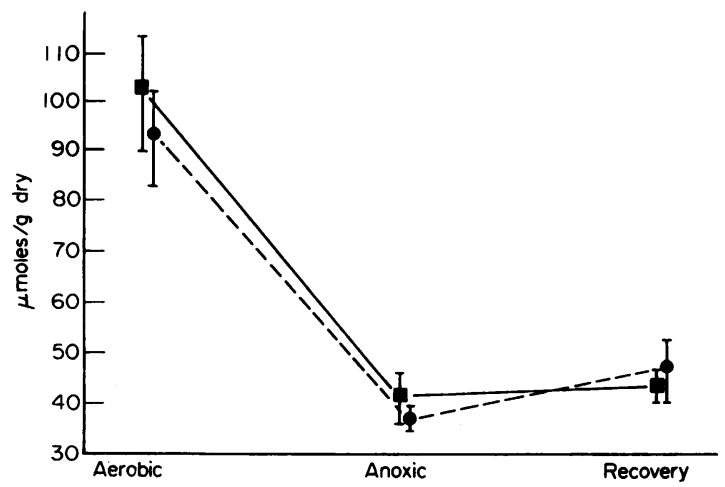

FIG. 3. Comparison of tissue glycogen levels with and without hyperosmolal mannitol. Glycogen concentration fell during anoxia and remained depressed during recovery. No significant differences between the two perfusates were seen. $\square, \mathrm{KRB}$; O, KRB + mannitol; $P=$ N.S.

\section{Tissue high energy phosphate stores}

Hyperosmolality did little to alter the depletion of myocardial high energy phosphate stores during anoxia nor its repletion during recovery (Fig. 4). Creatine phosphate levels of $28 \cdot 1 \pm 1 \cdot 4 \mu \mathrm{mol} / \mathrm{g}$ dry wt during isosmolar aerobic perfusion fell to negligible levels $(0.3 \mu \mathrm{mol})$ during anoxia and rose to $20 \cdot 6 \pm 3 \mu \mathrm{mol} / \mathrm{g}$ dry wt with recovery aerobic perfusion. Concentrations obtained with hyperosmolar buffer were (a) $29.4 \pm 6 \cdot 5$, (b) 0.2 , and (c) $24 \cdot 6 \pm 3 \mu \mathrm{mol} / \mathrm{g}$ dry wt; $P=$ N.S. (Fig. 4). Tissue ATP concentration showed a qualitatively similar change. With isosmolar buffer, $\mathrm{ATP}=$ (a) $20 \cdot 1 \pm 1 \cdot 3$, (b) $10 \cdot 1 \pm 1 \cdot 1$, (c) $8 \cdot 5 \pm 1 \cdot 2 \mu \mathrm{mol} / \mathrm{g}$ dry wt. Hyperosmolal perfusion did not significantly improve this balance nor did the presence of the hyperosmolal buffer significantly alter ADP, or AMP concentrations in the recovery phase.

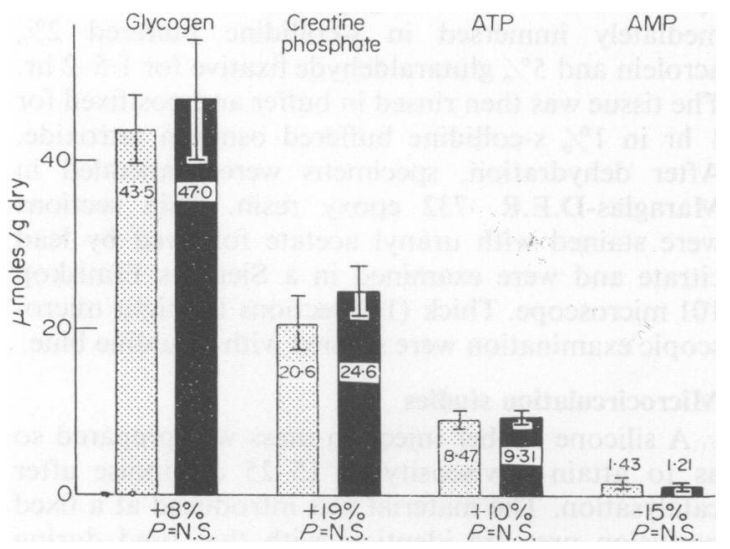

FIG. 4. Summary of tissue studies during recovery from anoxia. No difference in concentrations of glycogen, creatine phosphate, ATP or AMP were observed. Perfusate osmolality did not affect ADP levels (not illustrated). 䙵, KRB; $\mathbf{C}, \mathrm{KRB}+$ mannitol.

\section{Myocardial tissue water content}

Presence of a hyperosmolal perfusate significantly affected total tissue water content during all three phases of perfusion (Fig. 5). The tissue wet/dry wt ratio with isosmolar perfusion in period (a) was $6.68 \pm 0.41$ and rose to $7.48 \pm 0.56(P=<0.01)$ with anoxia. It remained elevated throughout the recovery period $(7 \cdot 55 \pm 0 \cdot 17)$. Hyperosmolal perfusion reduced the ratio during aerobic perfusion $(5 \cdot 80 \pm$ $0 \cdot 26$ ). Water content during anoxic perfusion was significantly less than that seen with isosmolal buffer and remained fixed during recovery (ratio $=6 \cdot 77 \pm$ $0 \cdot 19)$. In studies which utilized the isosmolal buffer during periods (a) and (b) and switched to the hyperosmolal buffer during (c) tissue wet/dry wt ratios was restored to control levels. 


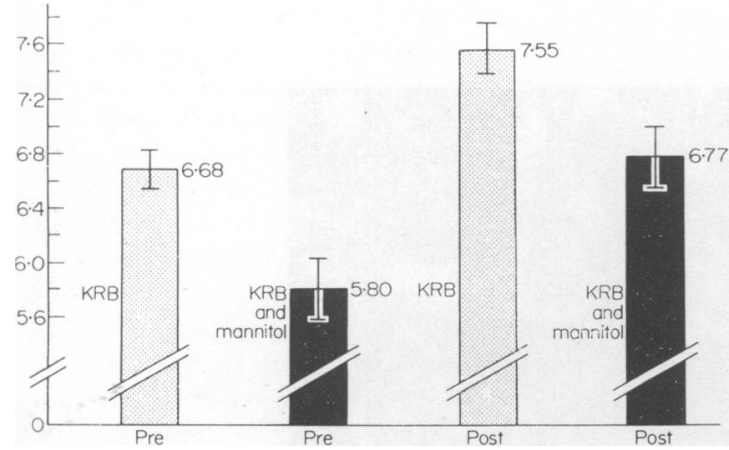

FIG. 5. Effect of mannitol on myocardial water content (wet wt/dry wt ratios). 'Pre'=aerobic period before anoxia. 'Post' = reoxygenated recovery period. Hyperosmolal perfusion reduces the ratio during the aerobic period. The increase in ratio noted during and following anoxia was prevented by an increase in osmolality.

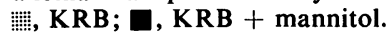

\section{Electron microscopic studies}

\section{(a) Control hearts}

Electron microscopic examination of rat myocardium fixed immediately after removal revealed a submicroscopic appearance which did not differ significantly from that of other mammals. Both isosmolal and hyperosmolal perfused tissue showed identical electron microscopic anatomy (Fig. 6).

\section{(b) Anoxic hearts}

Hearts subjected to a $15-\mathrm{min}$ anoxic period perfused with KRB alone showed the most severe sub- microscopic pathology. Marked disorganization of most of the myocardial cell constituents was evident. The mitochondrial matrix was extracted (electron-lucent configuration) and these organelles were randomly dispersed in the sarcoplasm. Some of the mitochondria were markedly swollen and contained fewer cristae. Elements of the sarcoplasmic reticulum were dilated and disrupted, and vacuoles of various sizes were also prominent (Fig. 7). In some junctional areas, the intercalated discs were disrupted, and dense bodies of unknown composition were seen in the sarcoplasm. Myofibrillar alterations were also noted. Normal sarcolemmal and subsarcolemmal structure was altered owing to disruption and osmotic swelling of myocytes. Focal endothelial cell changes included swelling and a reduction in the number of pinocytotic vesicles.

The presence of mannitol in the perfusate during the anoxic period significantly reduced the severity of these changes (Fig. 8). Approximately $50 \%$ of the mitochondria now remained in the condensed configuration and myofibrillar structure was generally maintained.

\section{(c) Reoxygenated hearts}

Reoxygenation of hearts perfused with KRB alone showed only minimal reversal of the submicroscopic alterations found in the anoxic hearts (Fig. 9). Most of the mitochondria examined continued to demonstrate an extracted, electron-lucent matrix, and various myofibrillar alterations such as fraying of myofilaments. The occurrence of contraction bands

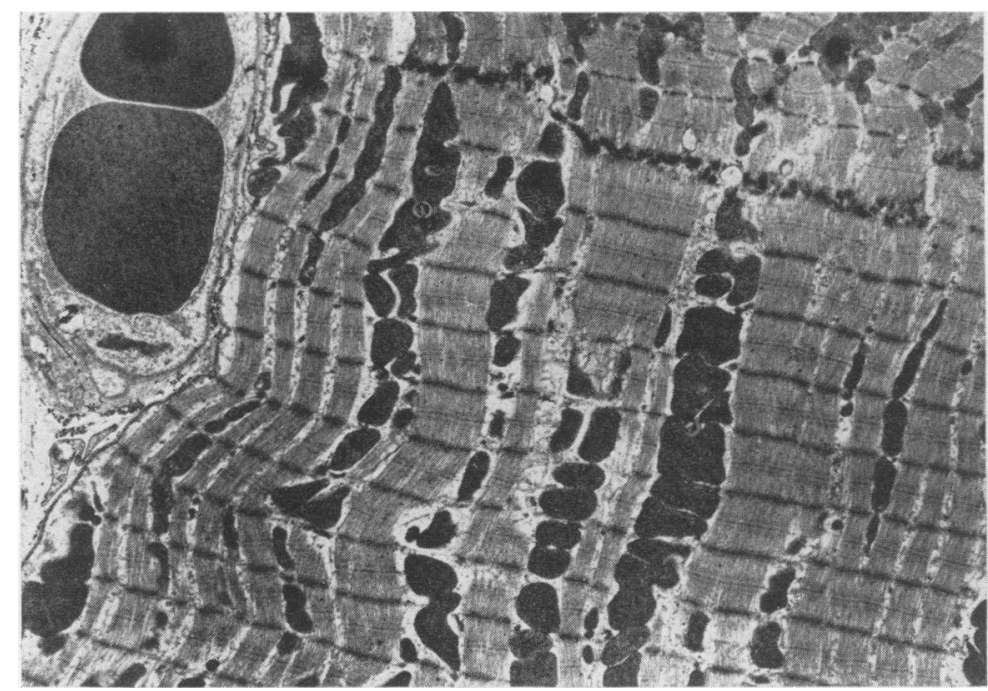

Fig. 6. Rat heart fixed immediately after removal (no perfusion). Note the small capillary and intercalated disc. $\times 7400$. 


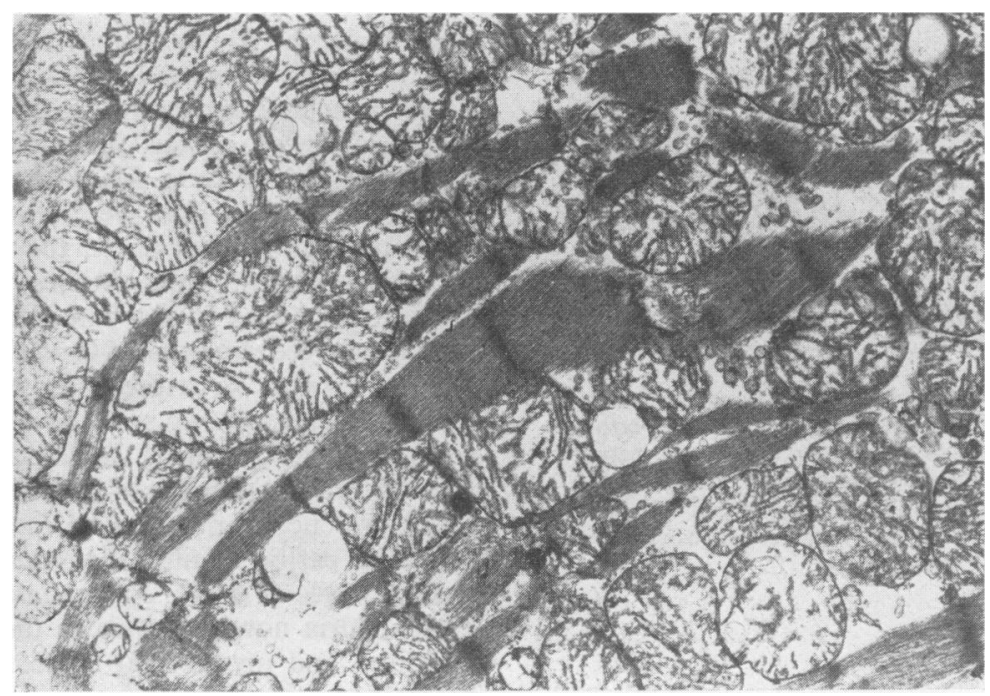

Fig. 7. Rat myocardium after $15 \mathrm{~min}$ anoxic perfusion with KRB. Note the disorganized, altered myofibrils and the electron-lucent mitochondria with disrupted cristae. $\times 15,000$.

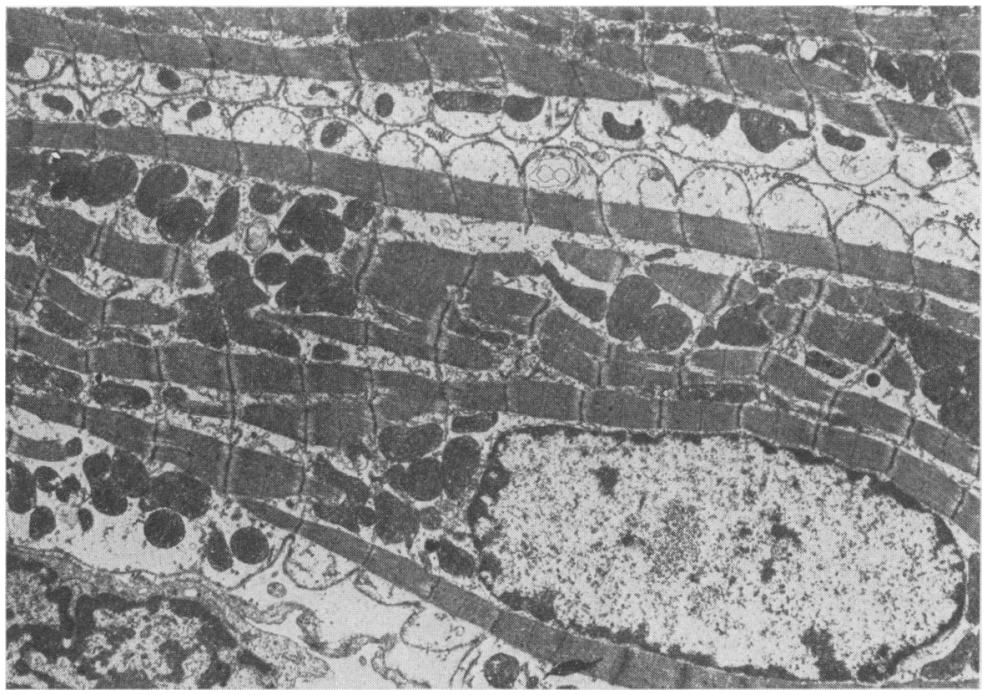

Fig. 8. Rat myocardium after $15 \mathrm{~min}$ anoxic perfusion with hyperosmolal medium. Note that myocardial cell constituents show only slight alteration. $\times 7400$. 


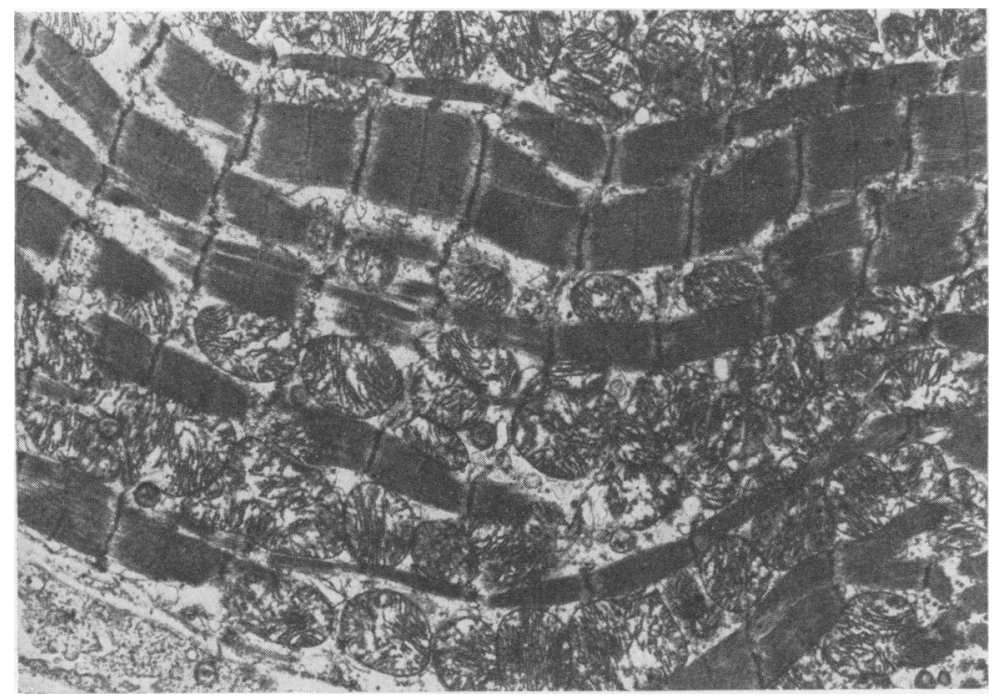

FIG. 9. Reoxygenated rat myocardium perfused with isosmolal KRB buffer. Large areas of subcellular damage are still evident. $\times 12,000$.

persisted despite reoxygenation. Focal areas of endothelial swelling and vacuolization were also apparent.

After reoxygenation with mannitol, a substantial reversal of the above mentioned anoxic changes was noted. Many of the myocardial and vascular endothelial cells were virtually identical in substructure to the controls (Fig. 10). Even in areas where contraction bands and myofibrillar fusion persisted, most of the mitochondria were in the condensed configuration. It should be pointed out, however, that scattered focal areas of severe, apparently irreversible, damage were still evident.

\section{Microcirculation studies}

The coronary microcirculation as studied by the silicone rubber injection technique visualized arteriolar and capillary vessels to a diameter of $7 \mu$. No significant areas of capillary obstruction were noted and no differential effect of mannitol on arteriolar or

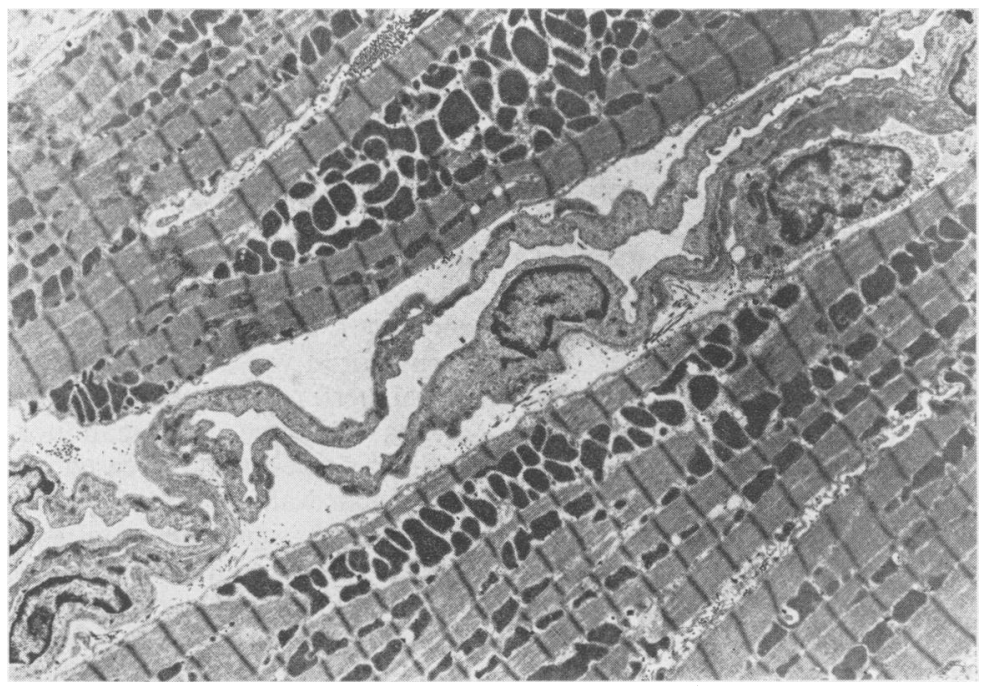

Fig. 10. Rat myocardium after $15 \mathrm{~min}$ of reoxygenated perfusion (recovery) with hyperosmolal medium. Note that both myocardial cells and capillaries show essentially normal ultrastructure. $\times 6000$. 
capillary filling was observed after anoxia or during the reoxygenated recovery period.

\section{Discussion}

Leaf (1970) has recently emphasized the physiological significance of intracellular volume regulation. His studies of the heart and experiments with kidney and brain have demonstrated that intracellular oedema may accentuate or prolong the effects of oxygen deprivation after the stress of a reduction in flow has been relieved. Willerson's studies (1972) in the post-coronary ligated dog heart demonstrated that elevated osmolality protected the ischaemic myocardium against the potentially toxic effects of increased cell volume. There was an improvement in left ventricular function curves, a lessening of ST segment elevation and an increase in total and collateral flow. Our studies have confirmed the positive inotropic effects of this agent when perfusate osmolality was raised by $60 \mathrm{mmol}$ either before or after the anoxic stress.

It seems probable that induced obligatory extracellular hyperosmolality may: improve regional perfusion by reducing endothelial cell swelling and increasing patency of arterioles and capillaries or by directly reducing coronary arteriolar resistance (Willerson et al., 1972); protect mitochondria by maintaining an optimal ultrastructural configuration for oxidative phosphorylation and energy production (Caulfield et al., 1972); enhance inotropism by a relative increase in $\mathrm{Ca}^{++}$concentration or an increase in $\mathrm{Ca}^{++}$activity from small molecular weight complexes (Caulfield, 1973). An increase in extracellular osmotic potential would aid in the maintenance of a favourable intracellular environment by counteracting swelling induced by ischaemia (Jennings, Herdson and Sommers, 1969) and exploit the inverse relationship between ventricular wall compliance and an abnormal increase in myocardial cell volume.

We were unable to demonstrate an increase in total coronary flow during hyperosmolal perfusion at either high or low $p \mathrm{O}_{2}$. In the absence of formed elements capillary entrapment was, of course, not possible. Furthermore, a reversal of capillary and arteriolar endothelial wall swelling sufficient to improve flow might well have been masked by the gross nature of coronary flow measurement utilized or by the marked arteriolar vasodilatation in response to anoxia. Silicone latex injection studies of the vascular tree performed and reported here also failed to demonstrate a difference in vessel calibre between these two types of buffer.

Ultrastructural alterations produced in mammalian myocardium following oxygen deprivation are well known and appear to be relatively independent of the means of inducing hypoxia. Mitochondria are extremely sensitive to anoxia and morphological changes in these structures were most striking in this study. We observed a loss of matrix constituents or a change from an electron-dense (condensed) configuration to an electron-lucent (extracted) configuration. Other mitochondrial alterations observed and also reported by other investigators included disappearance of intra-mitochondrial granules, swelling and fragmentation of cristae, increase in number and of size, and fusion. Severe mitochondrial changes induced by anoxia proved to be reversible during reoxygenated perfusion with hyperosmolal mannitol and were considerably reduced in myocardial samples taken immediately after anoxia. The degree of cellular oedema noted in isosmolal perfusates studied during anoxia was reduced by approximately $50 \%$ when buffer osmolality was elevated by addition of mannitol. Mannitol also appeared to decrease the amount of myofibrillar oedema, fusion, and contracture noted after anoxia. This protective effect correlated well with the enhanced haemodynamic recovery seen during the subsequent reoxygenation period.

The presence of mannitol in the perfusate did not significantly affect total coronary flow and no differences in arteriolar or capillary filling could be observed using the silicone rubber injection technique.

Although severe anoxic mitochondrial swelling was largely prevented during phases (b) and (c) of the hyperosmolal perfusion such histological improvement was not expressed biochemically by improvement in oxidative phosphorylation, enhanced utilization of glycogen or glucose, or maintenance or improvement in high energy phosphate stores.

Significant dissociation between preservation of mitochondrial and myofibrillar structure and their functional capability was evident in our model. The improvement in haemodynamic performance induced by hyperosmolality did not appear to be related to a similar improvement in myocardial energetics. There was a positive correlation between reduction in wet/dry wt ratios and enhanced mechanical performance during the improved recovery of phase (c) seen when mannitol was added. These findings suggest that increased compliance of the left ventricular wall due to a decrease in cell volume was of primary significance in explaining our data. Investigations continue in an effort to determine whether this simply represents a reversal of the detrimental effect of tissue fluid pools or a relative or absolute increase in intracellular $\mathrm{Ca}^{++}$concentration with enhanced inotropic response to this ion, already competing with increased $\mathrm{H}^{+}$ion for contractile binding sites during myocardial anoxia. 


\section{References}

Apstein, C.S., Puchner, E. \& Brachfeld, N. (1970) Improved automated lactate and simultaneous determination. In: Transactions of Technicon International Congress. Advances in Automated Analysis, 41.

Caulfield, J.B., Willerson, J.T., Weisfeldt, M.L. \& Powell, J. (1972) Effect of mannitol on hypoxic myocardium. Circulation, 45 (supplement II), 120.

Caulfield, J.B. (1973) An explanation for the positive inotropic effect of hypertonic solutions. Proceedings of the VI Annual Meeting of the International Study Group for Research in Cardiac Metabolism, Abstract \# 197.
Jennings, R.B., Herdson, P.B. \& Sommers, H.M. (1969) Structural and functional abnormalities in mitochondria isolated from ischemic dog myocardium. Laboratory Investigation, 20, 548.

LEAF, A. (1970) Regulation of intracellular fluid volume and disease. American Journal of Medicine, 49, 291.

Willerson, J.T., Powell, J., Guiney, T.E., Stark, J.J., SANDERS, C.A. \& LeAF, A. (1972) Improvement in myocardial function and coronary blood flow in ischemic myocardium after mannitol. Journal of Clinical Investigation, 51, 2989. 